\title{
Dynamics of a Two Subpopulations System Including Immigration
}

\author{
A. Zincenko, S. Petrovskii * \\ Department of Mathematics, University of Leicester, LE1 RH, UK
}

\begin{abstract}
The phenomenon of replacement migration into declining population prompts development of multicomponent models in population dynamics. We propose a simple model of population including resident and migrant components with migration flow as an external input. The main assumption is that offspring that are born to migrants will have the same vital rates as the resident population. The proposed model is based on partial differential equation to take into account the age structure of the population. The formulae for exact solutions are derived. We focus on the case when native population declines in the absence of migration. Assuming a sufficiently large constant growth rate of migration we obtain asymptotic solutions as $t \rightarrow \infty$. Using the asymptotic solutions, we have calculated "critical" value of growth rate of migrant inflow that is the value that provides, as time tends to infinity, the equal number of residents and migrants in the population. We provide numerical illustrations using demographic data for Germany in 2010.
\end{abstract}

Keywords and phrases: multicomponent model including immigration, McKendrick-von Foerster type model, critical value

Mathematics Subject Classification: 35Q91, 35Q92, 91D20

\section{Introduction}

Models of population dynamics play major roles in many areas of science such as human demography, ecology, and biology (Stott, 2011) and are integral to population management (Menges, 1990; Fujiwara and Caswell, 2001). There are two types of population growth models: continuous-time and continuousage models (Sharpe and Lotka, 1911; McKendrick., 1926; Von Foerster, 1959) and discrete time-variable and discrete-age scale model (Bernadelli, 1941; Leslie 1945). Keyfitz and Keyfitz (1997) compare the McKendrick-von Foerster equation with discrete (i.e. Leslie-type) models and show some advantages of the continuous model. One can consider Leslie matrix model as an approximation to McKendrick model. But this is nothing more than one of many possible approximation procedures. This approach lacks flexibility, which could lead to significant errors in projection. In particular, the discretization of age distribution in the boundary condition leads to errors, since the true projected value, in fact, depends on the way in which the population is distributed within the age intervals. Henson (1998) discusses the relationship between Leslie and the PDE models. She notes that Leslie model is often low-

\footnotetext{
${ }^{*}$ Corresponding author. E-mail: sp237@le.ac.uk
} 
dimensional, and therefore, is a too coarse discretization for the full implementation of the respective PDE. Below we illustrate a discrepancy between the results obtained for the same data in the framework of continuous and discrete models (see Section 6, Figures 7 and 8). The effect of migration on a population structure is studied less. Understanding the impact of human migration on population structure dynamics is important for several reasons as it is known to have a variety of economic, social, cultural and other implications. In particular, human travel in general and migration in particular are apparently responsible for the geographical spread of human infectious disease (Hufnagel et al., 2004). As a result of increased human mobility, the last two decades have seen outbreaks of several dangerous diseases. Swine influenza (Nicholson and Webster, 1998; Webby and Webster, 2003; Myers et al., 2007), Ebola fever (Goeijenbier et al., 2014) and sexually transmitted diseases (Grassly et al., 2005) are just a few examples of infection that is transmitted from human to human, so that their proliferation is directly affected by human travel. Therefore, studies of demographic implication of human travel is of fundamental importance.

Such studies are relevant in relation to the situation of population aging and decline, which occur in economically developed countries, such as Germany (United Nations Population Division, 2000). Without migration, the total population of Germany would have already started to decline (Ulrich, 1998). Since a sharp growth of birth rate is unlikely in Europe, the importance of migration as a source of population growth is obvious. Immigration also seems to be a possible way to overcome ageing of the native population of the developed countries. Some publications argue that immigration cannot solve this problem (Coale, 1986; Espenshade, 1994; Day, 1996; Bijak et al., 2008). However, the projections in these papers cover only about a 40-year period. It is necessary to assess whether population dynamics will reach stability during this period.

In this paper, we study a linear model of McKendrick-von Foerster type for the time development of an age structured two component female population including internal migration. However, in contrast to the McKendrick-von Foerster equation, our model is a system of two partial differential equations, which are connected with each other through a boundary condition on the line corresponding to the zero age. Our main demographic assumption is that immigrant-born offspring exhibit the same vital rates as local inhabitants (Alba and Nee (1997), Gordon (1964) and Carter (2000)).

\section{Model}

We study the McKendrick-von Foerster (McKendrick, 1926; Von Foerster, 1959) two subpopulations system of first order linear partial differential equations:

$$
\begin{aligned}
& \frac{\partial N_{1}}{\partial t}+\frac{\partial N_{1}}{\partial x}=-\mu_{1}(x, t) N_{1}, \\
& \frac{\partial N_{2}}{\partial t}+\frac{\partial N_{2}}{\partial x}=-\mu_{1}(x, t) N_{2}+p(x, t),
\end{aligned}
$$

where $(x, t) \in \Omega=\left\{(x, y) \in \mathbb{R}^{2} \mid x \in(0, X), t>0\right\}$, which is a differential form of the conservation law of the number of individuals in the continuous model of the age structure of a population that consists of two subpopulations with external immigration into the second subpopulation. Here $\mu_{1}, \mu_{2}, p$ are continous nonnegative functions, and $X$ is a positive number.

It is a dynamical model of a female population consisting of residents and migrants; $\mathrm{x}$ denotes the age of individuals, $t$ - the time, $N_{1}$ - the age density of residents, $N_{2}$ - the age density of migrants, $p$ - the migrant inflow age density, $\mu_{1}, \mu_{2}$ - the age specific mortality rates, and $X$ - the maximum lifespan of an individual.

The boundary $\partial \Omega$ of the region $\Omega$ is the union of three rays: $\partial \Omega=\Gamma_{1}+\Gamma_{2}+\Gamma_{3}$, where $\Gamma_{1}$ is the $x$ positive semiaxis, $\Gamma_{2}$ is the $t$ positive semiaxis, and $\Gamma_{3}=\left\{(x, t) \in \mathbb{R}^{2} \mid x=X, t \geq 0\right\}$. On $\Gamma_{1}$, we set the standard initial conditions:

$$
N_{1}(x, 0)=\psi_{1}(x), \quad N_{2}(x, 0)=\psi_{2}(x),
$$


and on $\Gamma_{2}$ we set the following conditions:

$$
\begin{aligned}
& N_{1}(0, t)=\int_{0}^{x} f_{1}(x, t) N_{1}(x, t)+f_{2}(x, t) N_{2}(x, t) \mathrm{d} x, \\
& N_{2}(0, t)=0 .
\end{aligned}
$$

Here $f_{1}$ and $f_{2}$ are non-negative continuous functions, which can be interpreted as age specific fertility rates. We assume that $f_{1}(X)=f_{2}(X)=0$. The formulae (2.3)-(2.4) mean that, in the model under consideration, the offspring born to the migrants belong to the resident subpopulation. Notice that one does not impose additional restrictions on values of functions $N_{1}$ and $N_{2}$ on $\Gamma_{3}$.

The characteristics of the equations (2.1) are the straight lines $x-t=$ const. Therefore, the influence region of the conditions (2.3) is $\Omega_{1}=\{(x, t) \in \Omega \mid x>t\}$, and the influence region of the conditions (2.4)-(2.5) is $\Omega_{2}=\{(x, t) \in \Omega \mid x<t\}$. In the region $\Omega_{1}$, the solution to the Cauchy problem (2.1)-(2.3) exists and is unique. The same is true for equation (2.2) with initial condition (2.5) in the region $\Omega_{2}$. In section 2, we give the solutions to these problems.

In Section 3, for the region $\Omega_{2}$, we reduce equation (2.1) with the additional condition (2.4) to a 2nd kind Volterra integral equation. We conclude that the solution of the problem exists, it is unique and can be efficiently computed by numerical methods.

Remark: if $\psi_{2}(0)>0$ then the solution will be discontinuous on the line $x=t$.

\section{Exact Solutions}

In the region $\Omega_{1}$, we represent the characteristics parametrically as follows:

$$
\begin{aligned}
x(\tau) & =x_{0}+\tau, \\
t(\tau) & =\tau,
\end{aligned}
$$

where $x_{0}$ is the abscissa of the intersection point of an characteristic with the $x$ axis. Then $x_{0}, \tau$ are new coordinates on $\Omega_{1}$. Moreover, clearly

$$
\begin{aligned}
& x_{0}=x-t, \\
& \tau=t .
\end{aligned}
$$

The functions $N_{1}$ and $N_{2}$ on a characteristic are the solutions to the following linear initial value problems:

$$
\begin{aligned}
& \frac{\mathrm{d} N_{1}}{\mathrm{~d} \tau}+\mu_{1}\left(x_{0}+\tau, \tau\right) N_{1}=0, \\
& N_{1}(0)=\psi_{1}\left(x_{0}\right), \\
& \frac{\mathrm{d} N_{2}}{\mathrm{~d} \tau}+\mu_{2}\left(x_{0}+\tau, \tau\right) N_{2}=p\left(x_{0}+\tau, \tau\right), \\
& N_{2}(0)=\psi_{2}\left(x_{0}\right) .
\end{aligned}
$$

Solving these problems and substituting the expressions (3.2) for the variables $x_{0}, \tau$, we find:

$$
\begin{aligned}
& N_{1}(x, t)=\psi_{1}(x-t) \exp \left\{-\int_{0}^{t} \mu_{1}(x-t+s, s) \mathrm{d} s\right\}, \\
& N_{2}(x, t)=\psi_{2}(x-t) \exp \left\{-\int_{0}^{t} \mu_{2}(x-t+s, s) \mathrm{d} s\right\}+\int_{0}^{t} p(x-t+k, k) \mathrm{e}^{-\int_{k}^{t} \mu_{2}(x-t+s, s) \mathrm{d} s} \mathrm{~d} k .
\end{aligned}
$$

In the region $\Omega_{2}$, we represent the characteristics parametrically as follows:

$$
\begin{aligned}
x(\tau) & =\tau, \\
t(\tau) & =t_{0}+\tau,
\end{aligned}
$$


The function $N_{2}$ on a characteristic is the solution to the following linear initial value problem:

$$
\begin{aligned}
& \frac{\mathrm{d} N_{2}}{\mathrm{~d} \tau}+\mu_{2}\left(x_{0}+\tau, \tau\right) N_{2}=p\left(x_{0}+\tau, \tau\right), \\
& N_{2}(0)=0 .
\end{aligned}
$$

Solving this Cauchy problem and returning to the variables $x, t$ we find that, if $(x, t) \in \Omega_{2}$, then

$$
N_{2}(x, t)=\int_{0}^{X} p(k, t-x+k) \mathrm{e}^{-\int_{k}^{x} \mu_{2}(s, t-x+s) \mathrm{d} s} \mathrm{~d} k .
$$

\section{Reducing problem (2.1), (2.4) to a 2nd kind Volterra integral equation}

We cannot write immediately the solution to equation (2.1) in the domain $\Omega_{2}$ as we do not know boundary function $N_{1}(0, t)$. We will do the following. Denote $N_{1}(0, t)=b(t)$. We can express function $N_{1}(x, t), t>x$ via $b(t)$ :

$$
N_{1}(x, t)=b(t-x) \mathrm{e}^{-\int_{0}^{x} \mu_{1}(s, t-x+s, s) \mathrm{d} s},
$$

According to condition (2.4), the function $b(t)$ conversely can be expressed via the solution to the problem (2.1)-(2.4):

$$
b(t)=\int_{0}^{X} f_{1}(x, t) N_{1}(x, t)+f_{2}(x, t) N_{2}(x, t) \mathrm{d} x .
$$

Substituting expression (4.1) into equation (4.2), we get the following integral equation:

$$
b(t)=f(t)+\int_{0}^{\min (t, X)} K(x, t) b(t-x) \mathrm{d} x,
$$

where

$$
f(t)=\int_{\min (t, X)}^{X} f_{1}(x, t) N_{1}(x, t) \mathrm{d} x+\int_{0}^{X} f_{2}(x, t) N_{2}(x, t) \mathrm{d} x
$$

and

$$
K(x, t)=f_{1}(x, t) \mathrm{e}^{-\int_{0}^{X} \mu_{1}(s, t-x+s) \mathrm{d} s}
$$

By changing integration variable $t-x=y$ in (4.3), we get the following integral equation for unknown function $b$ :

$$
b(t)=f(t)+\int_{t-\min (t, X)}^{t} K(t-y, t) b(y) \mathrm{d} y .
$$

Finally, by denoting

$$
L(t, y)= \begin{cases}K(t-y, t) & t-y<X \\ 0 & t-y>X\end{cases}
$$

we get the following 2 nd kind Volterra equation:

$$
b(t)=f(t)+\int_{0}^{t} L(t, y) b(y) \mathrm{d} y .
$$

The following result of the theory of Volterra equations is well known: for a continuous kernel and a continuous free term, there exists a continuous solution of the Volterra equation of the second kind, which is, moreover, unique (see Hazewinkel, 2001). This implies that there exists a unique solution to the problem (2.1)-(2.5). 


\section{An asymptotic solution}

In this section, we assume that birth and death rates do not depend on time.

Let $\lambda$ be the unique solution to the following equation:

$$
\int_{0}^{X} f_{1}(x) \mathrm{e}^{-\lambda x-\int_{0}^{x} \mu_{1}(y) \mathrm{d} y} \mathrm{~d} x=1 .
$$

Then, in the absence of migrants, $\lambda$ is the asymptotic growth rate of the solution to the problem (2.1), (2.3), (2.4) in the following sense: there exists the limit

$$
\lim _{t \rightarrow \infty} \mathrm{e}^{-\lambda t} N_{1}(x, t)
$$

in the norm of the space $L^{1}([0, X])$ (see Arino, Sánchez \& Bravo de la Parra, 1998).

Let us consider the case when migration flow grows exponentially and has a constant age structure:

$$
p(x, t)=p_{0}(x) \mathrm{e}^{k t},
$$

with $k>\lambda$. Then the growth rate $k$ of the migrant inflow $p$ is also the asymptotic growth of the population. That is, there exists $\lim _{t \rightarrow \infty} N_{1}(x, t) \mathrm{e}^{-k t}$ and $\lim _{t \rightarrow \infty} N_{2}(x, t) \mathrm{e}^{-k t}$. We briefly outline the proof. Let $t$ be greater than $X$. Then, using (5.3), formula (3.9) results in $N_{2}(x, t)=\mathrm{e}^{k t} N_{2}(x)$. Now (4.2) takes the following form:

$$
b(t)=\int_{0}^{X} f_{1}(x) \mathrm{e}^{-\int_{0}^{x} \mu_{1}(s) \mathrm{d} s} b(t-x) \mathrm{d} x+C \mathrm{e}^{k t}
$$

with some constant $C$. Representing the function $b(t)$ in the form $b(t)=\mathrm{e}^{k t} \mu(t)$, we arrive at the integral equation

$$
\mu(t)=\int_{0}^{X} f_{1}(x) \mathrm{e}^{-k t-\int_{0}^{x} \mu_{1}(s) \mathrm{d} s} \mu(t-x) \mathrm{d} x+C,
$$

which implies

$$
\mu^{\prime}(t)=\int_{0}^{X} f_{1}(x) \mathrm{e}^{-k t-\int_{0}^{x} \mu_{1}(s) \mathrm{d} s} \mu^{\prime}(t-x) \mathrm{d} x .
$$

Using (5.1), we get the inequality

$$
\left|\mu^{\prime}(t)\right| \leq \alpha \max _{x \in\{0, X\}}\left|\mu^{\prime}(t-x)\right|
$$

with some $\alpha<1$. It follows that $\lim _{t \rightarrow \infty} \mu^{\prime}(t)=0$. Therefore, there exists $\mu_{\infty}=\lim _{t \rightarrow \infty} \mu(t)=0$, which leads to

$$
\lim _{t \rightarrow \infty} b(t) \mathrm{e}^{-k t}=\mu_{\infty}
$$

We now use (4.1) to conclude that $N_{1}(x, t)$ takes the form $\mathrm{e}^{k t} n_{1}(x)$ as $t \rightarrow \infty$. We find the corresponding asymptotic (as $t \rightarrow \infty$ ) solution to the problem, which is nothing more than a solution of stable population growth:

$$
\begin{aligned}
& N_{1}(x, t)=n_{1}(x) \mathrm{e}^{k t} \\
& N_{2}(x, t)=n_{2}(x) \mathrm{e}^{k t}
\end{aligned}
$$


Substituting (5.4), (5.5) into (2.1), (2.2), (2.4), (2.5), taking into account (5.3), and canceling $\mathrm{e}^{k t}$, we get the following linear ODEs:

$$
\begin{aligned}
& n_{1}^{\prime}(x)+\left(k+\mu_{1}(x)\right) n_{1}(x)=0, \\
& n_{2}^{\prime}(x)+\left(k+\mu_{2}(x)\right) n_{1}(x)=p_{0}(x),
\end{aligned}
$$

with the conditions

$$
\begin{aligned}
& n_{1}(0)=\int_{0}^{X} f_{1}(x) n_{1}(x)+f_{2}(x) n_{2}(x) \mathrm{d} x, \\
& n_{2}(0)=0 .
\end{aligned}
$$

The solution to initial value problem $(5.7),(5.9)$ is as follows:

$$
n_{2}(x)=\int_{0}^{x} p_{0}(y) \mathrm{e}^{-\int_{y}^{x}\left(k+\mu_{2}(z) \mathrm{d} z\right.} \mathrm{d} y,
$$

and any solution to equation (5.6) is of the form

$$
n_{1}(x)=n_{1}(0) \mathrm{e}^{-\int_{0}^{x}\left(k+\mu_{1}(y) \mathrm{d} y\right.} .
$$

Therefore, using condition (5.8), we obtain the equation for the initial value $n_{1}(0)$ :

$$
n_{1}(0)=\int_{0}^{X} f_{1}(x) n_{1}(0) \mathrm{e}^{-\int_{0}^{x}\left(k+\mu_{1}(y)\right) \mathrm{d} y} \mathrm{~d} x+\int_{0}^{X} f_{2}(x) n_{2}(x) \mathrm{d} x,
$$

that results in the formula

$$
n_{1}(0)=\frac{\int_{0}^{X} f_{2}(x) n_{2}(x) \mathrm{d} x}{1-\int_{0}^{X} f_{1}(x) \mathrm{e}^{-\int_{0}^{x}\left(k+\mu_{1}(y)\right) \mathrm{d} y} \mathrm{~d} x} .
$$

Substituting (5.13) into (5.11), we finally get:

$$
n_{1}(x)=\frac{\int_{0}^{X} f_{2}(x) n_{2}(x) \mathrm{d} x}{1-\int_{0}^{X} f_{1}(x) \mathrm{e}^{-\int_{0}^{x}\left(k+\mu_{1}(y)\right) \mathrm{d} y} \mathrm{~d} x} \mathrm{e}^{-\int_{0}^{x}\left(k+\mu_{1}(y)\right) \mathrm{d} y} .
$$

\section{Numerical illustrations}

The calculations are based on the population of Germany. We use the Federal Statistical Office of Germany (Destatis) data from 2010 as initial data (an email demografie@destatis.de, personal communication, February 2015). Mortality and fertility rates for Germany, in the year 2010, were modelled as empirical formulae, using these data as represented below. The mortality rates for local inhabitants were modelled using the following formula:

$$
\mu_{1}(x)=\exp \left\{-11.8+0.106 x+\frac{20.4}{1.89+x}\right\} .
$$

The mortality rates for immigrants were modelled by the formula

$$
\mu_{2}(x)=\exp \left\{-11.7+0.105 x+\frac{19.255}{1.69+x}\right\} .
$$


The fertility rates are modelled using the Beta function of Hoem et al.(1981):

$$
f(x)=R \frac{\Gamma(A+B)}{\Gamma(A) \Gamma(B)}(\beta-\alpha)^{-(A+B-1)}(x-\alpha)^{A-1}(\beta-x)^{B-1}, \quad \text { for } \quad \alpha<x<\beta
$$

The parameters are related to the mean $\nu$ and the variance $\tau^{2}$ of the density through the relations

$$
B=\left(\frac{(\nu-\alpha)(\beta-\nu)}{\tau^{2}}\right) \frac{\beta-\nu}{\beta-\alpha}
$$

and

$$
A=B \frac{\nu-\alpha}{\beta-\nu}
$$

According to Hoem et al. (1981), the parameters $\alpha$ and $\beta$ are interpreted as the lower and upper age limits of fertility, respectively. The parameter $R$ determines the overall level of fertility.

As a result, the following formulae for local inhabitants and immigrants are obtained:

$$
\begin{aligned}
& f_{1}(x)=(x-15)^{3.39}(55-x)^{6} \cdot 2.22 \cdot 10^{-14}, \quad 15 \leq x \leq 55, \\
& f_{2}(x)=(x-15)^{2.13}(55-x)^{4.77} \cdot 3.47 \cdot 10^{-11}, \quad 15 \leq x \leq 55 .
\end{aligned}
$$

Figures 1-4 illustrate agreement of the raw data with the above formulae.

We use the following models of the 2010 year age distributions of female native and migrant populations in Germany:

$$
\begin{aligned}
\psi_{1}(x)= & \exp \left\{12.2-0.0142 x+0.00444 x^{2}-0.000188 x^{3}\right. \\
& \left.+3.34 \cdot 10^{-6} x^{4}-2.57 \cdot 10^{-8} x^{5}+6.43 \cdot 10^{-11} x^{6}\right\}, \\
\psi_{2}(x)= & 0.2 \exp \left\{11.1+0.0345 x+0.00583 x^{2}-0.000335 x^{3}\right. \\
& \left.+7.34 \cdot 10^{-6} x^{4}-7.44 \cdot 10^{-8} x^{5}+2.81 \cdot 10^{-10} x^{6}\right\} .
\end{aligned}
$$

We have modeled the age distribution of migration inflow in the year 2010 as follows:

$$
p_{0}(x)=\exp \left\{7.78-2.02 x^{0.5}+0.801 x-0.0161 x^{2}+0.0000995 x^{3}\right\}-350,
$$

where $x$ stands for age in years. This formula agrees with the statistical data in the sense that it gives the same number of immigrants as in each age group represented in statistical data. Figure 5 shows a comparison between the raw data of net immigration flow by age group and the integrals of the modeled age distribution $p_{0}(x)$.

We have calculated the solution to the partial differential system (2.1)-(2.2) with the initial and boundary conditions (2.3)-(2.5) using quadrature formulae and numerically solving the Volterra equation (4.8). Based on obtained results, we have calculated time dependence of the size of the local inhabitant population and migrant population. The result for migrant inflow growth rate $k=0.01$ is represented on the plot in Figure 6.

Further, we have calculated the solution to ODEs (5.6), (5.7), with conditions (5.8) and (5.9) using numeric integration for different $k$ in the interval [0,0.02]. On the basis of obtained results, we have calculated the dependence of the limiting fraction of migrants on the growth rate of migrant inflow $k$. The result is represented on the plot in Figure 7.

The Figure 8 represents the corresponding result obtained with the help of matrix projection. The calculations are based on the same data as in Figure 7 . 


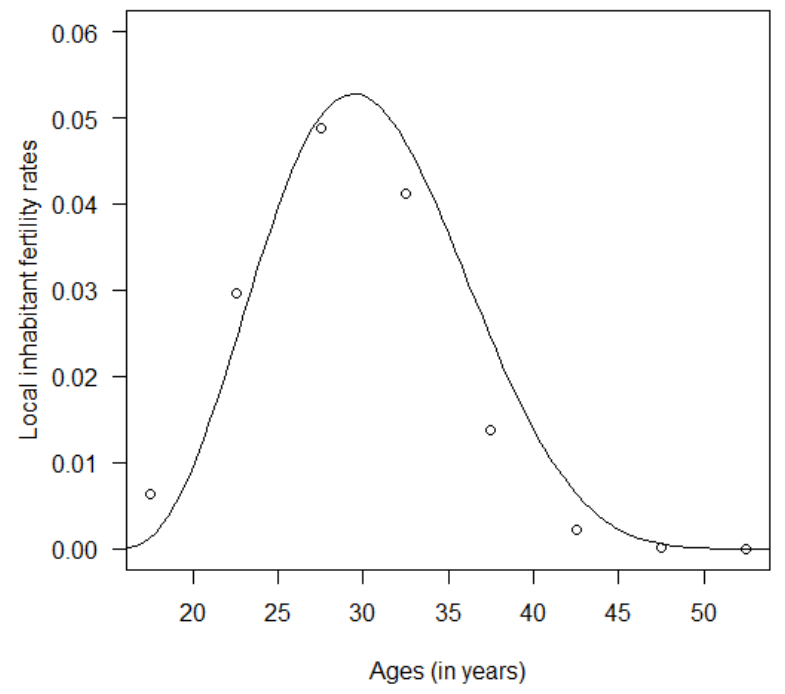

Figure 1. The line represents fertility rates calculated for local inhabitants of Germany, the year 2010 , calculated with the help of the formula (6.6).

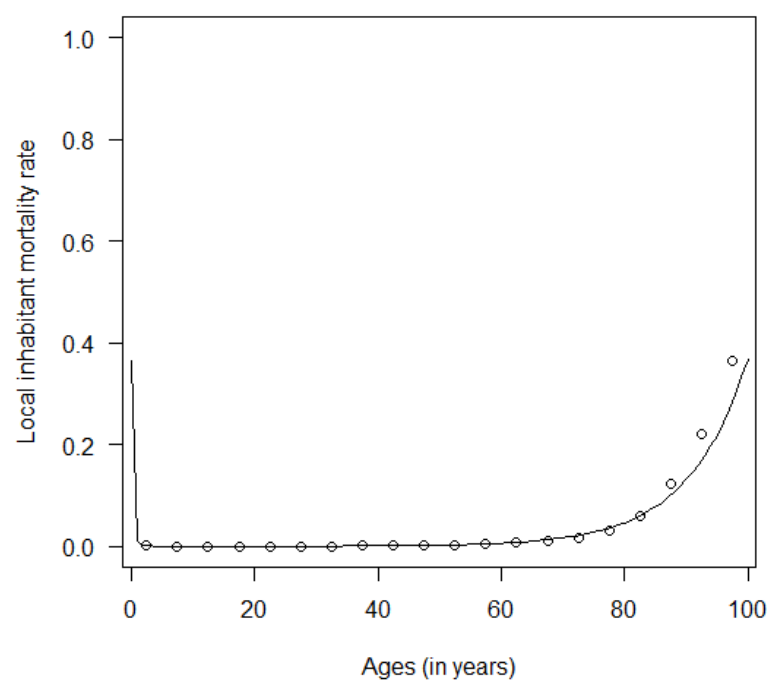

Figure 3. The line represents local inhabitants' mortality rates calculated with the help of the model $\log \mu(x)=a+b x+\frac{c}{d+x}$.

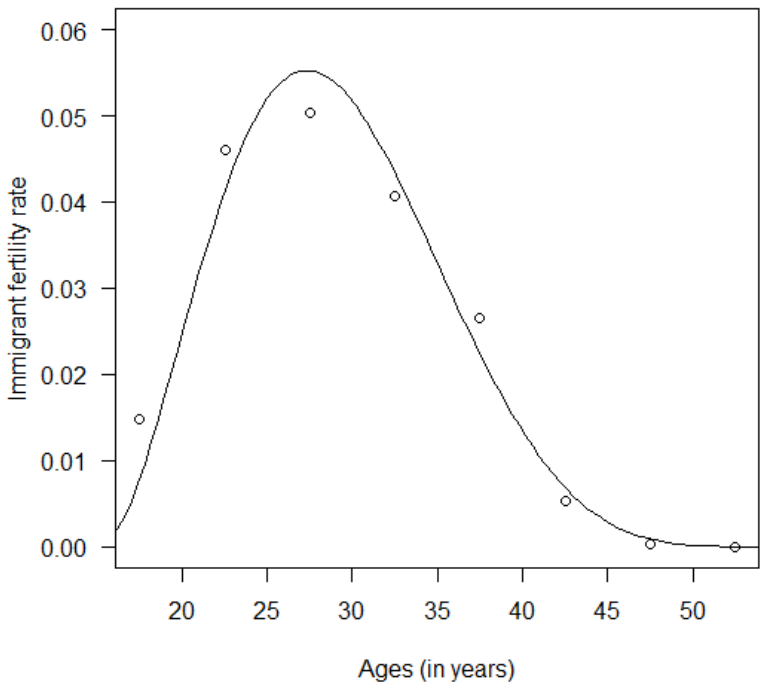

Figure 2. The line represents fertility rates calculated for immigrants of Germany, the year 2010, calculated with the help of the formula (6.7).

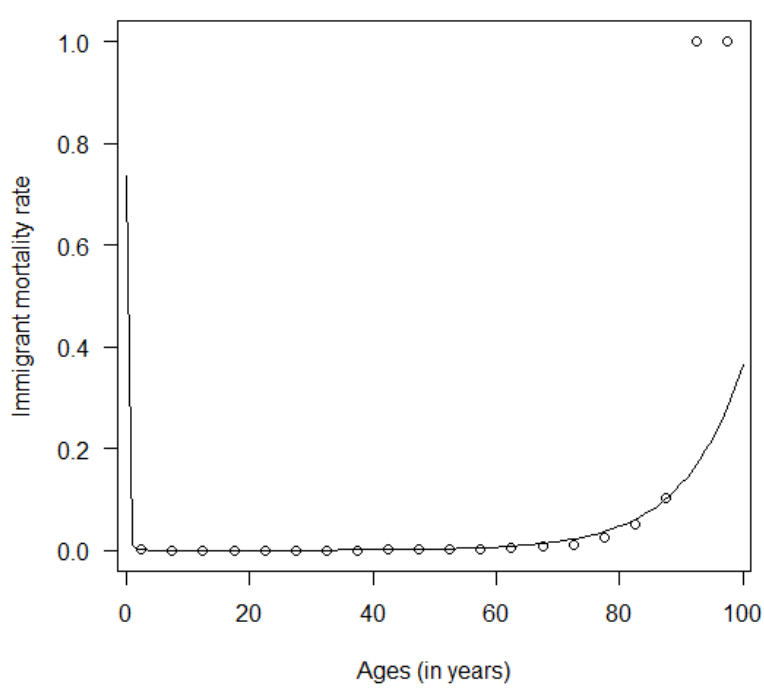

Figure 4. The line represents immigrants' mortality rates calculated with the help of the model $\log \mu(x)=a+b x+\frac{c}{d+x}$. 


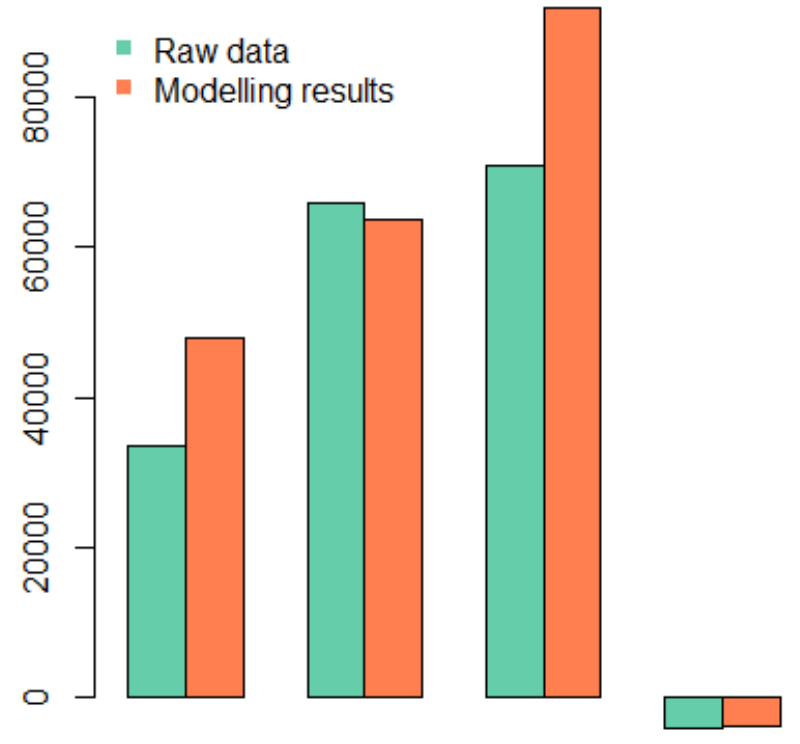

FiguRE 5. Net immigration flow for female-only population in Germany, year 2010, by the following age groups (in years): $0-18,18-25,25-50,50-65,65-100$. Modelling results were calculated as $\int_{x_{i}}^{x_{i+1}}=p_{0}(x) \mathrm{d} x$, where $x_{i}$ and $x_{i+1}$ are the bounds of the $i^{\text {th }}$ age group.

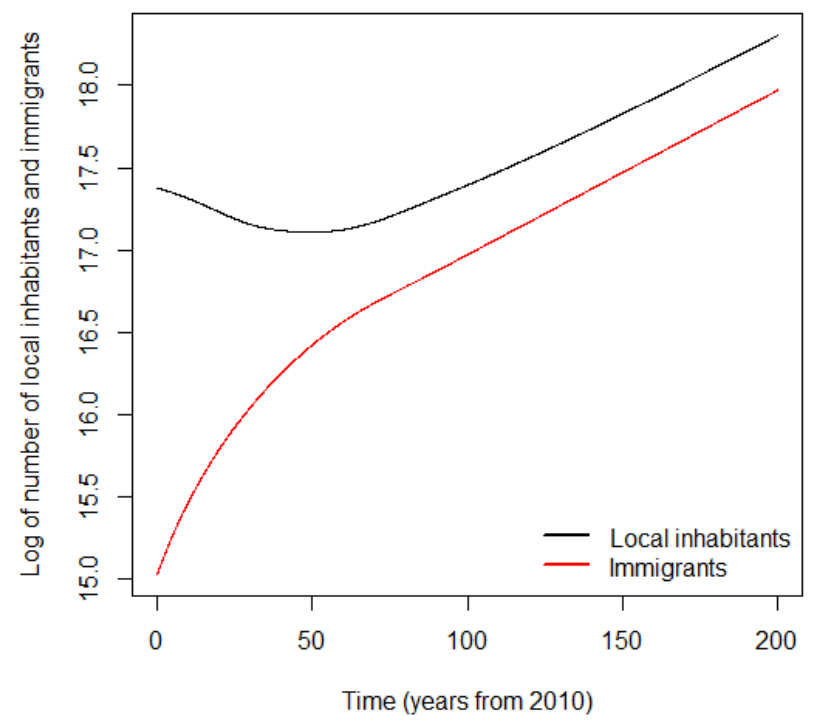

FiguRE 6. Logarithm of number of local inhabitants and immigrants projected over 200 years starting from 2010. Abundance was calculated using the model (2.1)-(2.5). Migration inflow is assumed to be of the form $p(x, t)=p_{0}(x) \mathrm{e}^{k t}$, with the initial age distribution of migrant inflow given by (6.10). Migrant inflow growth rate $(k)$ is set to 0.01 . When time is large, the growth rate of local inhabitants' abundance equals to that of immigrants' and the proportion of local inhabitants and immigrants stabilizes. 


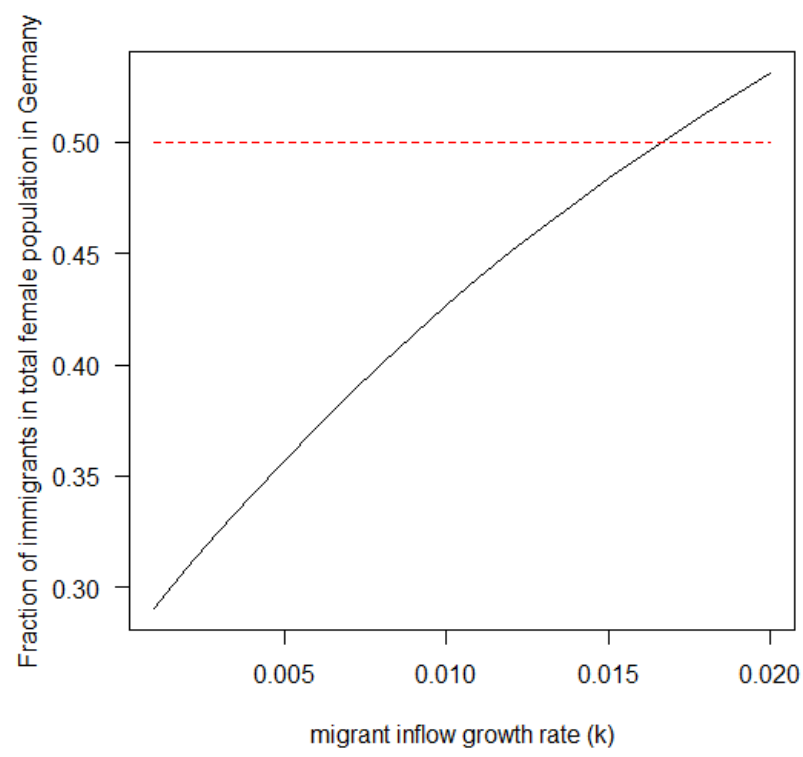

FiguRE 7. The limiting fraction of migrants in Germany's female population as a function of the migration growth rate $k$. The horizontal dashed line is a visual aid indicating $50 \%$ of migrants in the overall population. The crossover value $k=0.016$ is critical in the sense that faster growth of the migrant inflow leads to the predominance of migrants in the population.

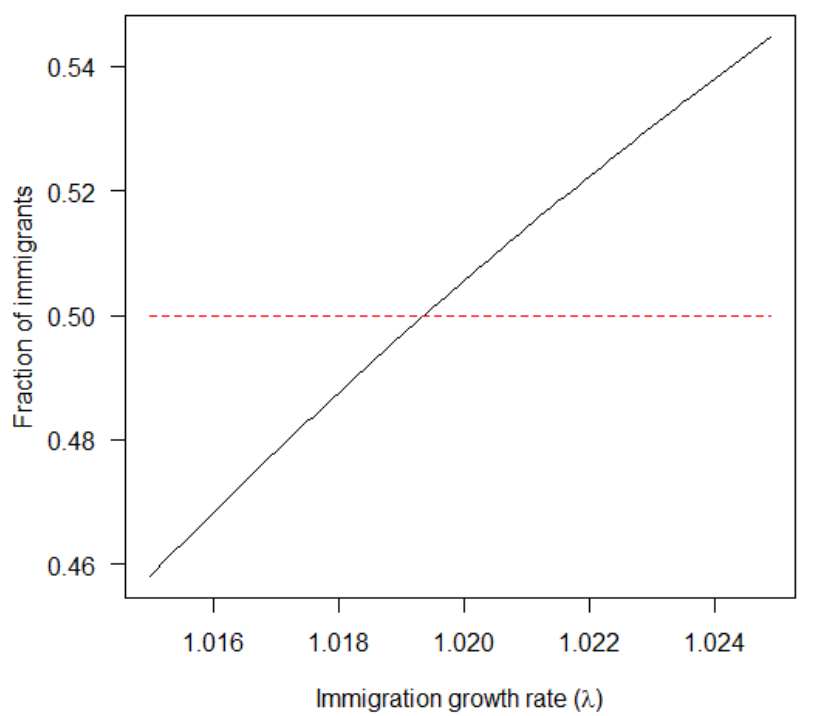

FiguRE 8. The limiting fraction of migrants in Germany's female population as a function of the migration growth rate $\lambda=\exp (k)$. The horizontal dashed line is a visual aid indicating $50 \%$ of migrants in the overall population. The crossover value $k=0.019$ is critical in the sense that faster growth of the migrant inflow leads to the predominance of migrants in the population. Results were obtained with the help of matrix projection. 


\section{Discussion and Conclusion}

We suggest a simple two-component population model including immigration as external input that is continuous in age and in time, and derive formulae to effectively investigate and calculate the solution to the corresponding mathematical problem. The main assumption is that the offspring born to the migrants belong to the resident subpopulation. It is, of course, a simplistic assumption as there are differences in fertility rates between children born to immigrants and residents. These differences are less pronounced for offspring that are born to couples where only one parent is an immigrant. Nevertheless, an assumption made in this work is reasonable as a first approximation.

Mathematically the model is a system of two linear first order partial differential equations that are connected only through the boundary condition. The boundary conditions are not classical, namely, boundary values of the unknown function are expressed through an integral operator acting on the same unknown function. As a numerical illustration, projecting of the Germany population is carried out under exponentially growing immigrant flow, and dependence of limiting proportion of migrants from the growth rate of the migrant inflow. Real data was used for 2010 as initial data. In the frame of our model, the stabilization time for the population dynamics is approximately 70 years. Calculations have shown that ageing in Germany would be offset during this time period with the help of a non-decreasing migration flow if the age-structure of the flow is as in 2010. Moreover, we have found that if the growth rate of migration flow is sufficiently small then the fraction of migrants in the population of Germany will be acceptable (if migration flow would increase by $1.6 \%$ per year then the limiting fraction of migrants in the population of Germany would be $50 \%$ ). The results are of practical relevance. Policy-makers can set sustainable immigration levels to offset population decline and ageing in the long-term. A notable outcome of this work is the framework developed that allows different immigration growth rates to be set, as well as different age structures of incoming migrants, thus enabling policy-makers to manage the evolution of the population.

Acknowledgements. A.Z. acknowledges the support of the Wellcome Trust (103780). A.Z. would like to thank T.H.G. Ezard, J. Bijak and S. Townley for useful discussions and comments.

\section{References}

[1] R. D Alba, V. Nee. Rethinking assimilation theory for a new era of immigrants. International Migration Review, 31 (1997), 826-874.

[2] O. Arino, E. Sanchez, R. Bravo de la Parra. A model of an age-structured population in a multipatch environment. Mathematical and Computer Modelling, 27 (1998), 137-150.

[3] H. Bernardelli. Population waves. Journal of the Burma Research Society, 31 (1941), 1-18.

[4] J. Bijak, D. Kupiszewska, M. Kupiszewski. Replacement migration revisited: Simulations of the effects of selected population and labour market strategies for the aging europe, 2002-2052. Population Research Policy Review, 27 (2008), 321-342.

[5] M. Carter. Fertility of mexican immigrant women in the U.S.: a closer look. Social Science Quarterly, 81 (2000), 404-420.

[6] A.J. Coale. Demographic effects of below replacement fertility and their social implications. In: Below replacement fertility in industrial societies: causes, consequences, policies (K. Davis, M.S. Bernstam, R. Ricardo-Campbell, Eds.) Cambridge University Press, Cambridge UK, 1986, pp.203-216.

[7] J. Day. Population projections of the united states, by age, sex, race, and hispanic origin: 1992 to 2050. Current Population Reports, 1996.

[8] T.J. Espenshade. Does the threat of border apprehension deter undocumented U.S. migration? Population and Development Review, 20 (1994), 871-892.

[9] H. Von Foerster. Some remarks on changing populations. In: The kinetics of cell proliferation (F. Stohlman, Jr, Ed.). Grune and Stratton, New York, 1959, pp.382-407.

[10] M. Fujiwara, H. Caswell. Demography of the endangered north atlantic right whale. Nature, 414 (2001), 537-541.

[11] M. Goeijenbier, J.J. van Kampen, C.B. Reusken, M.P. Koopmans, E.C. van Gorp. Ebola virusdisease: a review on epidemiology, symptoms, treatment and pathogenesis. The Nertherlands Journal of Medicine, 72 (2014), $442-448$.

[12] M. Gordon. Assimilation in American Life: The Role of Race, Religion, and National Origins. Wiley, London, 1965.

[13] N.C. Grassly, C. Fraser, G.P. Garnett. Host immunity and synchronized epidemics of syphilis across the United States. Nature, 433 (2005), 417-421. 
[14] M. Hazewinkel. Volterra equation. Encyclopedia of Mathematics. Kluwer, New York, 2001.

[15] S.M. Henson. Leslie matrix models as "stroboscopic snapshots" of McKendrick PDE models. Journal of Mathematical Biology, 37 (1998), 309-328.

[16] J.M. Hoem, D. Madsen, J.L. Nielsen, E. Ohlsen, H.O. Hansen, B. Rennermalm. Experiments in modelling recent Danish fertility curves. Demography, 18 (1981), 231-244.

[17] L. Hufnagel, D. Brockmann, T. Geisel. Forecast and control of epidemics in a globalized world. Proceedings of the National Academy of Sciences of the United States of America, 101 (2004), 15124-15129.

[18] B.L. Keyfitz, N. Keyfitz. The McKendrick Partial Differential Equation and Its Uses in Epidemiology and Population Study. Mathematical Computation Modelling, 26 (1997), 1-9.

[19] P.H. Leslie. On the use of matrices in certain population mathematics. Biometrika, 33 (1945), 183-212.

[20] A.G. McKendrick. Applications of mathematics to medical problems. Proceedings of the Edinburgh Mathematical Society, 44 (1926), 98-130.

[21] E.S. Menges. Population viability analysis for an endangered plant. Conservation Biology, 4 (1990), 52-62.

[22] K.P. Myers, C.W. Olsen, G.C. Gray. Cases of swine in uenza in humans: A review of the literature. Clinical Infectious Diseases, 44 (2007), 1084-1088.

[23] K. Nicholson, R.G. Webster. Textbook of Influenza. Blackwell, London, 1998.

[24] F.R. Sharpe, A.J. Lotka. A problem in age distribution. Philosophical Magazine, 21 (1911), 435-438.

[25] I. Stott. A framework for studying transient dynamics of population projection matrix models. Ecology Letters, 14 (2011), 959-970.

[26] R. Ulrich. Grau oder bunt? Zuwanderung und Deutschlands Bevölkerung im Jahre 2030. In: Migration und Gesundheit (T. Borde, M. David, H. Kentenich, Eds.). Mabuseverlag, Frankfurt, 1998, pp.17-32.

[27] United Nations Population Division. Replacement migration: Is it a solution to declining and ageing populations? 2001, http://www.un.org/esa/population/publications/.

[28] R.J. Webby, R.G. Webster. Are we ready for pandemic in uenza. Science, 302 (2003), 1519-1522. 